\title{
Prognostic Significance of the Lung Immune Prognostic Index in Patients with Resected Esophageal Squamous Cell Carcinoma
}

This article was published in the following Dove Press journal: Cancer Management and Research

\author{
Ji-Feng Feng ${ }^{1,2}$ \\ Jian-Ming Zhao ${ }^{3}$ \\ Sheng Chen' \\ Qi-Xun Chen ${ }^{1,2}$
}

'Department of Thoracic Oncological Surgery, Institute of Cancer Research and Basic Medical Sciences of Chinese Academy of Sciences, Cancer Hospital of University of Chinese Academy of Sciences, Zhejiang Cancer Hospital, Hangzhou, 310022, People's Republic of China; ${ }^{2}$ Key Laboratory Diagnosis and Treatment Technology on Thoracic Oncology, Hangzhou, 310022, People's Republic of China; ${ }^{3}$ Department of Thoracic Surgery, Jinhua Guangfu Hospital, Jinghua, 32100I, People's Republic of China
Correspondence: Ji-Feng Feng Department of Thoracic Oncological Surgery, Institute of Cancer Research and Basic Medical Sciences of Chinese Academy of Sciences, Cancer Hospital of University of Chinese Academy of Sciences, Zhejiang Cancer Hospital, No. 38 Guangji Road, Hangzhou, 310022, People's Republic of China Email Fengjf@zjcc.org.cn
Background: The lung immune prognostic index (LIPI), a novel index combined with serum lactate dehydrogenase (LDH) and derived neutrophil to lymphocyte ratio (dNLR), is recently proposed to predict prognosis in lung cancer. The LIPI is not a unique indicator for lung cancer. However, the prognostic role of LIPI has not yet been evaluated in extrapulmonary cancer. The aim of this study was to determine whether LIPI is still a useful prognostic indicator for patients with resected esophageal squamous cell carcinoma (ESCC). Methods: The clinical data including preoperative laboratory results for 361 consecutive resected ESCC cases from 2007 to 2010 were retrospectively analyzed. A LIPI based on serum LDH and dNLR was conducted, characterizing into 3 groups (LIPI 0, 1 and 2). The association between LIPI and cancer-specific survival (CSS) was analyzed according to the Kaplan-Meier method and Cox regression analysis with hazard ratio (HR) and 95\% confidence interval (CI). A nomogram model was conducted by R 3.6.0 software.

Results: In this study, 220 (60.9\%), 100 (27.7\%) and 41 (11.4\%) patients had a LIPI of 0, 1 and 2, respectively. The 5-year CSS for LIPI 0,1 and 2 was $40.9 \%, 19.0 \%$ and $9.8 \%$, respectively $(P<0.001)$. Subgroup analysis based on TNM stage revealed that HALP was also significantly related to CSS in any stage (TNM I: $P=0.002$; TNM II: $P=0.009$; TNM III: $P=0.031)$. The LIPI serves as an independent predictor regarding CSS in multivariate analyses in patients with resected ESCC. Compared to LIPI 0, LIPI 1 and 2 had an HR of 1.419 (95\% CI: $1.063-1.895, P=0.018)$ and 2.064 (95\% CI: $1.403-3.036, P<0.001)$ regarding CSS, respectively. A nomogram was also developed in individualized CSS prediction based on LIPI in patients with resected ESCC.

Conclusion: To the best of our knowledge, the present study is the first study to explore the association between LIPI and prognosis in patients with extra-pulmonary cancer. The LIPI, combined with LDH and dNLR, is still a potential independent prognostic marker in patients with resected ESCC.

Keywords: esophageal squamous cell carcinoma, lung immune prognostic index, lactate dehydrogenase, derived neutrophil to lymphocyte ratio, prognosis

\section{Introduction}

Esophageal cancer (EC) is one of the most usual cancers worldwide. ${ }^{1}$ As a major pathological type of EC in China, esophageal squamous cell carcinoma (ESCC) is the $3 \mathrm{rd}$ of incidence and the 4 th of mortality. ${ }^{2}$ ESCC remains a fatal disease because it is usually not detected until it has progressed to an advanced stage. Despite the management and treatment is improved in recent years, the prognosis for ESCC remains poor. ${ }^{3,4}$ According to the poor prognosis and high incidence, therefore, the 
detection and prediction methods prior to treatment for patients with ESCC are essential.

There are close relations between inflammation and cancer prognosis. ${ }^{5}$ Mantovani et $\mathrm{al}^{5}$ demonstrated that inflammation has many tumor-promoting effects, such as subverting adaptive immune responses, promoting metastasis and angiogenesis. An increasing number of studies in recent years have shown that inflammatory indexes, such as albumin (ALB), C-reactive protein (CRP), derived neutrophil to lymphocyte ratio (dNLR) and neutrophil to lymphocyte ratio (NLR) are associated with prognosis in a variety

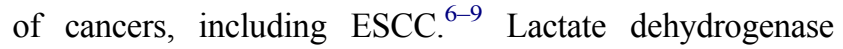
(LDH) plays a significant role in the tumor metastasis and proliferation. ${ }^{10,11}$ Moreover, pretreatment LDH correlates with prognosis of many malignant diseases, such as breast cancer, ${ }^{12}$ hepatocellular carcinoma, ${ }^{13}$ and melanoma. ${ }^{14}$ However, the association between LDH and prognosis remains controversial in patients with ESCC. ${ }^{15-17}$

These indexes have the advantage of providing objective diagnostic results, which may potentially benefit clinicians and patients for their treatment. However, every single index might be affected by various factors. Therefore, the potential bias could be decreased by the combination of these indexes. Recently, the lung immune prognostic index (LIPI), combined with LDH and dNLR, was firstly reported by Mezquita et $\mathrm{al}^{18}$ in patients with metastatic non-small cell lung cancer (NSCLC). The results revealed that LIPI was associated with patients treated with immune checkpoint inhibitors. After that, more and more studies have been reported the association between LIPI and prognosis in NSCLC and small cell lung cancer (SCLC). ${ }^{19-22}$ LIPI is derived based on two factors for prognosis that are inexpensive and readily available in daily clinical practice: dNLR and LDH. The LIPI is not a unique prognostic indicator for lung cancer. To the best of our knowledge, the clinical significance of LIPI has never been investigated in patients with extra-pulmonary cancer. Therefore, the aim of the present study was to explore whether LIPI is still a useful prognostic marker for patients with resected ESCC. Moreover, this study also aimed to develop a nomogram to predict the personalized cancer-specific survival (CSS) prediction of patients with ESCC.

\section{Materials and Methods}

\section{Ethics Statement}

The present study was based on the Declaration of Helsinki and approved by the ethics committee of
Zhejiang Cancer Hospital (No. 2018-130). The requirement of patients' consent was waived by the ethics committee because the current study was retrospective and all data analyzed were anonymous.

\section{Patient Selection}

From January 2007 to December 2012, 361 consecutive cases with ESCC undergoing curative esophagectomy with lymphadenectomy were retrospectively analyzed. Patients were included in the present study according to the following inclusion criteria: (1) patients with resected stage I-III ESCC was confirmed, (2) patients with radical resection was conducted, (3) patients without any neoadjuvant treatment, (4) patients without any distant metastasis, and (5) clinical data including preoperative laboratory results were obtained before surgery within one week. The TNM stage was carried out in this study according to the 7th AJCC/ UICC TNM staging system. ${ }^{23}$

\section{Data Collection}

The main clinical data including preoperative laboratory results were collected according to the medical records. The laboratory results including ALB, LDH, CRP, white blood cell count and absolute neutrophil count were obtained within one week before surgery. The dNLR was defined as absolute neutrophil count to (white blood cell count-absolute neutrophil count) ratio. According to the previous studies, the LIPI is calculated by the serum LDH and dNLR, characterizing into 3 groups (LIPI 0,1 and 2, respectively). ${ }^{18}$ The cut-off value for dNLR was 3.0, and cut-off for LDH was defined according to the limit of each center (240 U/L for our hospital). In short, patients with both elevated dNLR $(>3.0)$ and LDH $(>240 \mathrm{U} / \mathrm{L})$ were assigned to a score of 2. Patients with one or no abnormal value were assigned to 1 or 0 , respectively. The cut-off values for ALB and CRP were $35.0 \mathrm{~g} / \mathrm{Land} 10.0 \mathrm{mg} / \mathrm{L}$, respectively.

\section{Statistical Analysis}

The chi-squared tests were used to evaluate the correlations grouped by LIPI. The association between LIPI and CSS was analyzed by the Kaplan-Meier method and Cox regression analysis with hazard ratio (HR) and $95 \%$ confidence interval (CI). Variables with statistical differences in univariate analyses were then subjected to multivariate analyses to identify independent prognostic factors by using a forward stepwise. Based on the multivariate analysis results, a prognostic nomogram was also established 
Table I Clinical Characteristics Based on LIPI in Patients with ESCC

\begin{tabular}{|c|c|c|c|c|c|}
\hline & Total $(n, \%)$ & LIPI 0 (n, \%) & LIPI I (n, \%) & LIPI $2(n, \%)$ & P-value \\
\hline Age (years) & & & & & 0.107 \\
\hline$\leq 60$ & $203(56.2)$ & $114(51.8)$ & $63(63.0)$ & $26(63.4)$ & \\
\hline$>60$ & $158(43.8)$ & $106(48.2)$ & $37(37.0)$ & $15(36.6)$ & \\
\hline Gender & & & & & 0.147 \\
\hline Male & $282(78.1)$ & $166(75.5)$ & $85(85.0)$ & $31(75.6)$ & \\
\hline Female & $79(21.9)$ & $54(24.5)$ & $15(15.0)$ & $10(24.4)$ & \\
\hline Tumor length $(\mathrm{cm})$ & & & & & 0.002 \\
\hline$\leq 3.0$ & $106(29.4)$ & $79(35.9)$ & $17(17.0)$ & $10(24.4)$ & \\
\hline$>3.0$ & $255(70.6)$ & |4I (64.1) & $83(83.0)$ & $31(75.6)$ & \\
\hline Tumor location & & & & & 0.906 \\
\hline Upper & $25(6.9)$ & $14(6.4)$ & $7(7.0)$ & $4(9.8)$ & \\
\hline Middle & $167(46.3)$ & $100(45.4)$ & $47(47.0)$ & $20(48.8)$ & \\
\hline Lower & $169(46.8)$ & $106(48.2)$ & $46(46.0)$ & $17(41.4)$ & \\
\hline Vessel invasion & & & & & 0.153 \\
\hline Negative & $301(83.4)$ & $190(86.4)$ & $78(78.0)$ & $33(80.5)$ & \\
\hline Positive & $60(16.6)$ & $30(13.6)$ & $22(22.0)$ & $8(19.5)$ & \\
\hline Perineural invasion & & & & & 0.091 \\
\hline Negative & $285(78.9)$ & $181(82.3)$ & $76(76.0)$ & $28(68.3)$ & \\
\hline Positive & $76(21.1)$ & $39(17.7)$ & $24(24.0)$ & $13(31.7)$ & \\
\hline Smoking & & & & & 0.664 \\
\hline No & $196(54.3)$ & $117(53.2)$ & $58(58.0)$ & $21(51.2)$ & \\
\hline Yes & $165(45.7)$ & $103(46.8)$ & $42(42.0)$ & $20(48.8)$ & \\
\hline Drinking & & & & & 0.575 \\
\hline No & $221(61.2)$ & $130(59.1)$ & $65(65.0)$ & $26(63.4)$ & \\
\hline Yes & $140(38.8)$ & $90(40.9)$ & $35(35.0)$ & $15(36.6)$ & \\
\hline Differentiation & & & & & 0.449 \\
\hline Well & $51(14.1)$ & $34(15.5)$ & $11(11.0)$ & $6(14.6)$ & \\
\hline Moderate & $240(66.5)$ & $149(67.7)$ & $67(67.0)$ & $24(58.5)$ & \\
\hline Poor & $70(19.4)$ & $37(16.8)$ & $22(22.0)$ & II (26.9) & \\
\hline TNM stage & & & & & $<0.001$ \\
\hline 1 & $93(25.8)$ & $70(31.8)$ & $17(17.0)$ & $6(14.6)$ & \\
\hline$\|$ & $115(31.9)$ & $80(36.4)$ & $25(25.0)$ & $10(24.4)$ & \\
\hline III & $153(42.3)$ & $70(31.8)$ & $58(58.0)$ & $25(61.0)$ & \\
\hline $\mathrm{dNLR}$ & & & & & $<0.001$ \\
\hline$\leq 3.0$ & $263(72.9)$ & $220(100.0)$ & $43(43.0)$ & $0(0.0)$ & \\
\hline$>3.0$ & $98(27.1)$ & $0(0.0)$ & $57(57.0)$ & $41(100.0)$ & \\
\hline LDH (U/L) & & & & & $<0.001$ \\
\hline$\leq 240$ & $278(77.0)$ & $220(100.0)$ & $58(58.0)$ & $0(0.0)$ & \\
\hline$>240$ & $83(23.0)$ & $0(0.0)$ & $42(42.0)$ & $41(41.0)$ & \\
\hline ALB $(g / L)$ & & & & & 0.013 \\
\hline$>35$ & $297(82.3)$ & $190(86.4)$ & $79(79.0)$ & $28(68.3)$ & \\
\hline$\leq 35$ & $64(17.7)$ & $30(13.6)$ & $21(21.0)$ & $13(31.7)$ & \\
\hline $\operatorname{CRP}(\mathrm{mg} / \mathrm{L})$ & & & & & 0.003 \\
\hline$\leq 10$ & $270(74.8)$ & $174(79.1)$ & $74(74.0)$ & $22(53.7)$ & \\
\hline$>10$ & $91(25.2)$ & $46(20.9)$ & $26(26.0)$ & $19(46.3)$ & \\
\hline
\end{tabular}

Abbreviations: ESCC, esophageal squamous cell carcinoma; LIPI, lung immune prognostic index; CRP, C-reactive protein; ALB, albumin; LDH, lactate dehydrogenase; $\mathrm{dNLR}$, derived neutrophil to lymphocyte ratio; TNM, tumor node metastasis; SD, standard deviation. 
by using Cox proportional hazard model. A nomogram model was conducted by R 3.6.0 software (Institute for Statistics and Mathematics, Vienna, Austria). ${ }^{24}$ Statistical analyses were performed with SPSS 20.0 (SPSS Inc., Chicago, IL, USA). All statistical analyses were twosided, and $P$ values less than 0.05 were considered statistically significant.

\section{Results}

\section{Patient Characteristics}

The patients included in this study consisted of 282 (78.1\%) men and 79 (21.9\%) women. The distribution of pathological TNM stages was as follows: TNM I 93 patients (25.8\%); TNM II 115 patients (31.9\%); and TNM III 153 patients (42.3\%) (Table 1). Figure 1A and B showed the histograms of the dNLR and LDH. Figure $1 \mathrm{C}$ shows the positive correlations between dNLR and LDH ( $\mathrm{r}=0.128, P=0.015)$. In this study, 220 (60.9\%), 100 $(27.7 \%)$ and $41(11.4 \%)$ patients had a LIPI of 0,1 and 2 , respectively. The clinical characteristics grouped by LIPI are shown in Table 1.

\section{CSS Analyses}

The 5-year CSS was $31.3 \%$ in the current study. The 5-year CSS for LIPI 0,1 and 2 were $40.9 \%, 19.0 \%$ and $9.8 \%$, respectively $(P<0.001$, Figure $2 \mathrm{~A})$. Figure 2B$\mathrm{D}$ demonstrated 5-year CSS curves which were stratified in subgroup analyses according to TNM stage in resected ESCC. According to different TNM stage, the results revealed that LIPI was also significantly related to CSS. In the group of TNM stage I, the 5-year CSS for LIPI 0, 1 and 2 were $57.1 \%$, $23.5 \%$ and $16.7 \%$, respectively $(P=0.002)$. In the group of TNM stage II, the 5-year CSS for LIPI 0, 1 and 2 were $40.0 \%$, $24.0 \%$ and $10.0 \%$, respectively $(P=0.009)$. In the group of TNM stage III, the 5-year CSS for LIPI 0, 1 and 2 were $25.7 \%$, $15.5 \%$ and $8.0 \%$, respectively $(P=0.031)$. Significantly differences were also found in the current study in patients grouped by dNLR ( $36.9 \%$ vs. $16.3 \%, P<0.001$, Figure $2 \mathrm{E})$ and LDH ( $36.7 \%$ vs. $13.3 \%, P<0.001$, Figure $2 \mathrm{~F}$ ).

\section{Univariate and Multivariate Analyses}

Following univariate analyses, it is recognized that CSS was significantly associated with TNM stage, perineural invasion, vessel invasion, tumor length, CRP, ALB, dNLR,
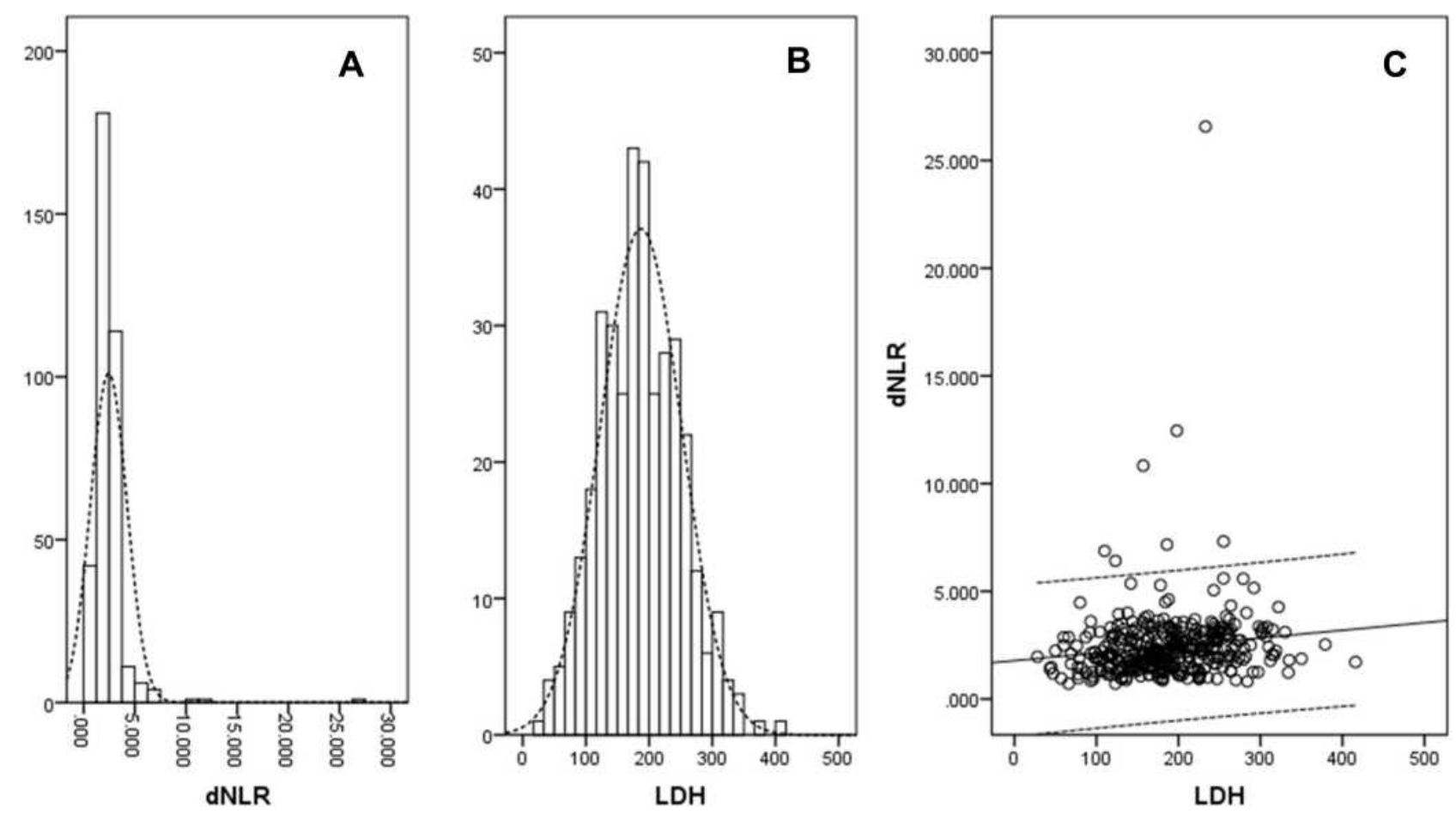

Figure I Histograms and correlation diagram.

Notes: The histograms of the $\operatorname{dNLR}(\mathbf{A})$ and LDH (B). Positive correlations between dNLR and LDH $(r=0.128, P=0.015)(\mathbf{C})$.

Abbreviations: $\mathrm{dNLR}$, derived neutrophil to lymphocyte ratio; $\mathrm{LDH}$, lactate dehydrogenase. 

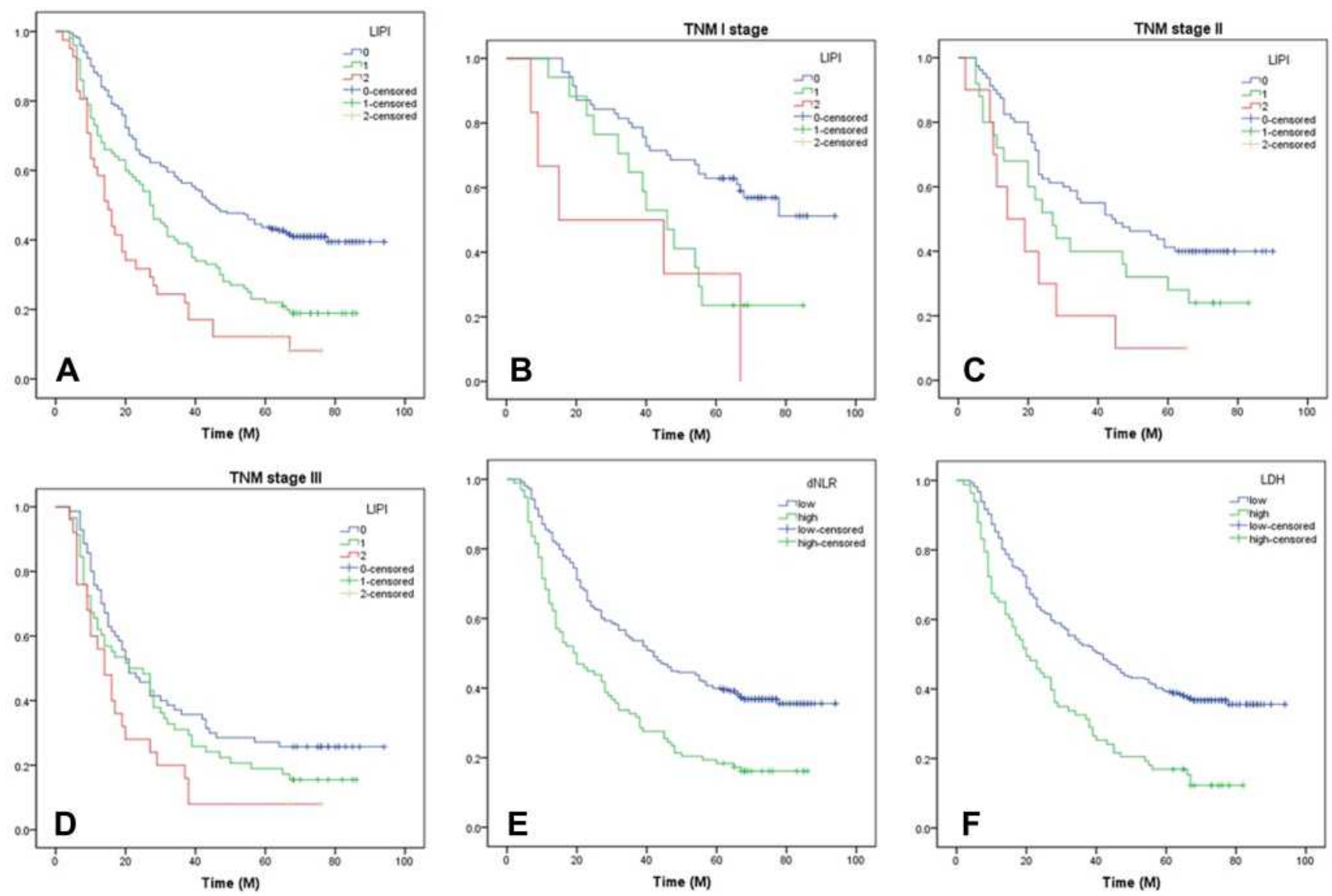

Figure 2 Kaplan-Meier curves for CSS stratified according to LIPI, dNLR and LDH.

Notes: Curves grouped by LIPI (The 5-year CSS for LIPI 0, I and 2 were $40.9 \%, 19.0 \%$ and $9.8 \%$, respectively, $P<0.00$ I) (A). Curves grouped by LIPI in subgroup analyses based on TNM (TNM I: $P=0.002$; TNM II, $P=0.009$; TNM III, $P=0.03$ I) (B-D). Curves grouped by dNLR $(36.9 \%$ vs. $16.3 \%, P<0.00 I)(E)$ and LDH (36.7\% vs. I3.3\%, $P<0.00 I)$ (F).

Abbreviations: LIPI, lung immune prognostic index; LDH, lactate dehydrogenase; CSS, cancer-specific survival; dNLR, derived neutrophil to lymphocyte ratio; TNM, tumor node metastasis.

LDH and LIPI (Table 2). Then, the above factors with significant differences in univariate analyses were included in multivariate analyses to identify independent prognostic factors by using a forward stepwise. In multivariate analyses, we found that LIPI $(P=0.001)$, instead of dNLR or $\mathrm{LDH}$, was an independent prognostic marker regarding CSS (Table 2). The LIPI serves as an independent predictor regarding CSS in multivariate analyses in patients with resected ESCC. Compared to LIPI 0, LIPI 1 and 2 had a HR of 1.419 (95\% CI: $1.063-1.895, P=0.018)$ and 2.064 (95\% CI: $1.403-3.036, P<0.001)$ regarding CSS, respectively. Moreover, TNM stage $(P<0.001)$ and CRP $(P=0.001)$ were also significant prognostic factors in this study (Table 2).

\section{Nomogram Analyses}

A nomogram prognostic model was also developed for LIPI in patients with resected ESCC. It would be helpful for us in individualized CSS survival prediction and better treatment. The significant prognostic factors in multivariate analyses, including LIPI, CRP and TNM stage, were included in nomogram model to predict the 1-, 3- and 5-year CSS probability for patients with resected ESCC (Figure 3).

\section{Discussion}

This study demonstrated that LIPI, instead of dNLR or LDH, serves as a useful independent prognostic marker. Therefore, the LIPI is not only a marker for patients in lung cancer, but also a useful prognostic marker for patients with resected ESCC. To the best of our knowledge, the present study is the first study to investigate the prognostic value of LIPI (combined with dNLR and LDH) in patients with resected ESCC.

The LIPI, combined with LDH and dNLR, was firstly reported by Mezquita et $\mathrm{al}^{18}$ in patients treated with 
Table 2 Univariate and Multivariate Analyses of CSS in ESCC Patients

\begin{tabular}{|c|c|c|c|c|}
\hline & \multirow{2}{*}{$\begin{array}{l}\text { Univariate Analysis } \\
\text { HR }(95 \% \mathrm{Cl})\end{array}$} & \multirow[t]{2}{*}{ P-value } & \multirow{2}{*}{$\begin{array}{l}\text { Multivariate Analysis } \\
\text { HR }(95 \% \mathrm{CI})\end{array}$} & \multirow[t]{2}{*}{ P-value } \\
\hline & & & & \\
\hline Age (years) & & 0.805 & & \\
\hline$\leq 60$ & 1.000 & & & \\
\hline$>60$ & $1.032(0.803-1.327)$ & & & \\
\hline Gender & & 0.757 & & \\
\hline Female & 1.000 & & & \\
\hline Male & I.050 (0.773-I.425) & & & \\
\hline Tumor length $(\mathrm{cm})$ & & 0.005 & & \\
\hline$\leq 3.0$ & 1.000 & & & \\
\hline$>3.0$ & $1.509(1.135-2.006)$ & & & \\
\hline Tumor location & & 0.423 & & \\
\hline Upper & 1.000 & & & \\
\hline Middle & I.449 (0.833-2.523) & 0.189 & & \\
\hline Lower & I.396 (0.802-2.43I) & 0.239 & & \\
\hline Vessel invasion & & 0.012 & & \\
\hline Negative & 1.000 & & & \\
\hline Positive & $1.496(1.09 I-2.052)$ & & & \\
\hline Perineural invasion & & 0.013 & & \\
\hline Negative & 1.000 & & & \\
\hline Positive & $1.446(1.1080-1.936)$ & & & \\
\hline Smoking & & 0.376 & & \\
\hline No & 1.000 & & & \\
\hline Yes & I.I I 9 (0.872-I.436) & & & \\
\hline Drinking & & 0.611 & & \\
\hline No & 1.000 & & & \\
\hline Yes & I.069 (0.827-I.382) & & & \\
\hline Differentiation & & 0.195 & & \\
\hline Well & 1.000 & & & \\
\hline Moderate & I.068 (0.73I-I.559) & 0.734 & & \\
\hline Poor & $1.397(0.898-2.174)$ & 0.138 & & \\
\hline TNM stage & & $<0.001$ & & $<0.001$ \\
\hline I & 1.000 & & 1.000 & \\
\hline II & $1.655(1.153-2.376)$ & 0.006 & $1.543(1.072-2.219)$ & 0.019 \\
\hline III & $2.719(1.944-3.802)$ & $<0.001$ & $2.303(1.633-3.248)$ & $<0.001$ \\
\hline CRP (mg/L) & & $<0.001$ & & 0.001 \\
\hline$\leq 10.0$ & 1.000 & & 1.000 & \\
\hline$>10.0$ & $1.977(1.508-2.593)$ & & $1.627(1.228-2.155)$ & \\
\hline ALB $(g / L)$ & & $<0.001$ & & \\
\hline$>35.0$ & 1.000 & & & \\
\hline$\leq 35.0$ & $1.943(1.433-2.634)$ & & & \\
\hline $\mathrm{dNLR}$ & & $<0.001$ & & \\
\hline$\leq 3.0$ & 1.000 & & & \\
\hline$>3.0$ & $1.903(1.459-2.483)$ & & & \\
\hline
\end{tabular}

(Continued) 
Table 2 (Continued).

\begin{tabular}{|c|c|c|c|c|}
\hline & \multirow{2}{*}{$\begin{array}{l}\text { Univariate Analysis } \\
\text { HR }(95 \% \mathrm{Cl})\end{array}$} & \multirow[t]{2}{*}{ P-value } & Multivariate Analysis & \multirow[t]{2}{*}{ P-value } \\
\hline & & & HR (95\% Cl) & \\
\hline LDH (U/L) & & $<0.001$ & & \\
\hline$\leq 240$ & 1.000 & & & \\
\hline$>240$ & $1.976(1.500-2.604)$ & & & \\
\hline LIPI & & $<0.001$ & & 0.001 \\
\hline 0 & 1.000 & & 1.000 & \\
\hline I & $1.776(1.345-2.346)$ & $<0.001$ & $1.419(1.063-1.895)$ & 0.018 \\
\hline 2 & $2.874(1.987-4.157)$ & $<0.001$ & $2.064(1.403-3.036)$ & $<0.001$ \\
\hline Adjuvant therapy & & 0.275 & & \\
\hline No & 1.000 & & & \\
\hline Yes & $1.162(0.888-1.521)$ & & & \\
\hline
\end{tabular}

Abbreviations: ESCC, esophageal squamous cell carcinoma; LIPI, lung immune prognostic index; CSS, cancer-specific survival; CRP, C-reactive protein; ALB, albumin; LDH, lactate dehydrogenase; dNLR, derived neutrophil to lymphocyte ratio; TNM, tumor node metastasis; $\mathrm{Cl}$, confidence interval; HR, hazard ratio.

immune checkpoint inhibitors in metastatic NSCLC. After that, more and more studies have been reported the association between LIPI and prognosis in NSCLC and SCLC. ${ }^{19-22}$ Minami et al $^{19}$ reported that LIPI was an independent prognostic factor in patients treated with chemotherapy for adenocarcinoma with wild-type epidermal growth factor receptor (EGFR). The results revealed that LIPI was also associated with the prognosis in patients treated with EGFR-TKI therapy for mutant EGFR. A study including 11 clinical trials (5 studies for immune checkpoint inhibitor and 6 studies for TKI therapy) was performed by Kazandjian et $\mathrm{al}^{20}$ for metastatic NSCLC. The result showed that the LIPI score was associated with progression-free survival (PFS) and OS. Sonehara et $\mathrm{al}^{21}$ demonstrated that LIPI is an independent prognostic factor associated with OS in a retrospective study that enrolled 171 patients diagnosed with SCLC. All the previous studies revealed that LIPI was associated with prognosis in NSCLC and SCLC. LIPI is not a unique prognostic indicator for lung cancer. However, as far as we know, the clinical significance of LIPI has never been investigated in patients with ESCC.

It is well accepted that the dNLR and LDH are both common clinical biomarkers in daily clinical practice. It is well known that systematic inflammatory response is responsible for the poor prognosis in patients with cancers, including EC. ${ }^{6-9}$ Moreover, pretreatment LDH correlates with prognosis of many malignant diseases. ${ }^{12-14}$ However, the prognostic significance regarding serum LDH is still controversial in patients with ESCC. ${ }^{15-17}$ A retrospective study including 906 patients with ESCC reported that LDH (cut-off value: $361.5 \mathrm{U} / \mathrm{L}$ ) was associated with OS. ${ }^{15}$ However, another study regarding 212 patients revealed that LDH (cut-off value: $170 \mathrm{U} / \mathrm{L}$ ) was not associated with OS or PFS in patients with ESCC undergoing chemoradiotherapy. ${ }^{16}$ The results were consistent with another study which demonstrated that LDH (cut-off value: $154.4 \mathrm{U} / \mathrm{L}$ ) was not associated with $\mathrm{OS}$ in a retrospective study with ESCC. ${ }^{17}$

The nomogram has been recognized as a reliable tool for creating a simple and intuitive statistical predictive model to quantify the risk of clinical events. Therefore, more and more nomograms have been conducted in various cancers, some of which have been found to be more reliable than traditional staging system in recent years. Compared with the previous nomogram, ${ }^{25}$ our model only included three factors: LIPI, TNM stage and CRP. Other factors such as tumor length and differentiation are not independent factors in our study. It is worth mentioning that TNM stage was integrated in our nomogram model, the results were consistent with another published study reported by Zhang et al. ${ }^{26}$ Our nomogram also included the inflammation-based prognostic scores (LIPI and CRP). It is well accepted that close relations between inflammation and cancer prognosis. Recently, Shao et $\mathrm{al}^{27}$ and Zhang et $\mathrm{al}^{26}$ built a nomogram based on CRP to ALB ratio for ESCC, respectively. Although the ratio of CRP to ALB was not analyzed in the current study, CRP was integrated in the model. In addition, 


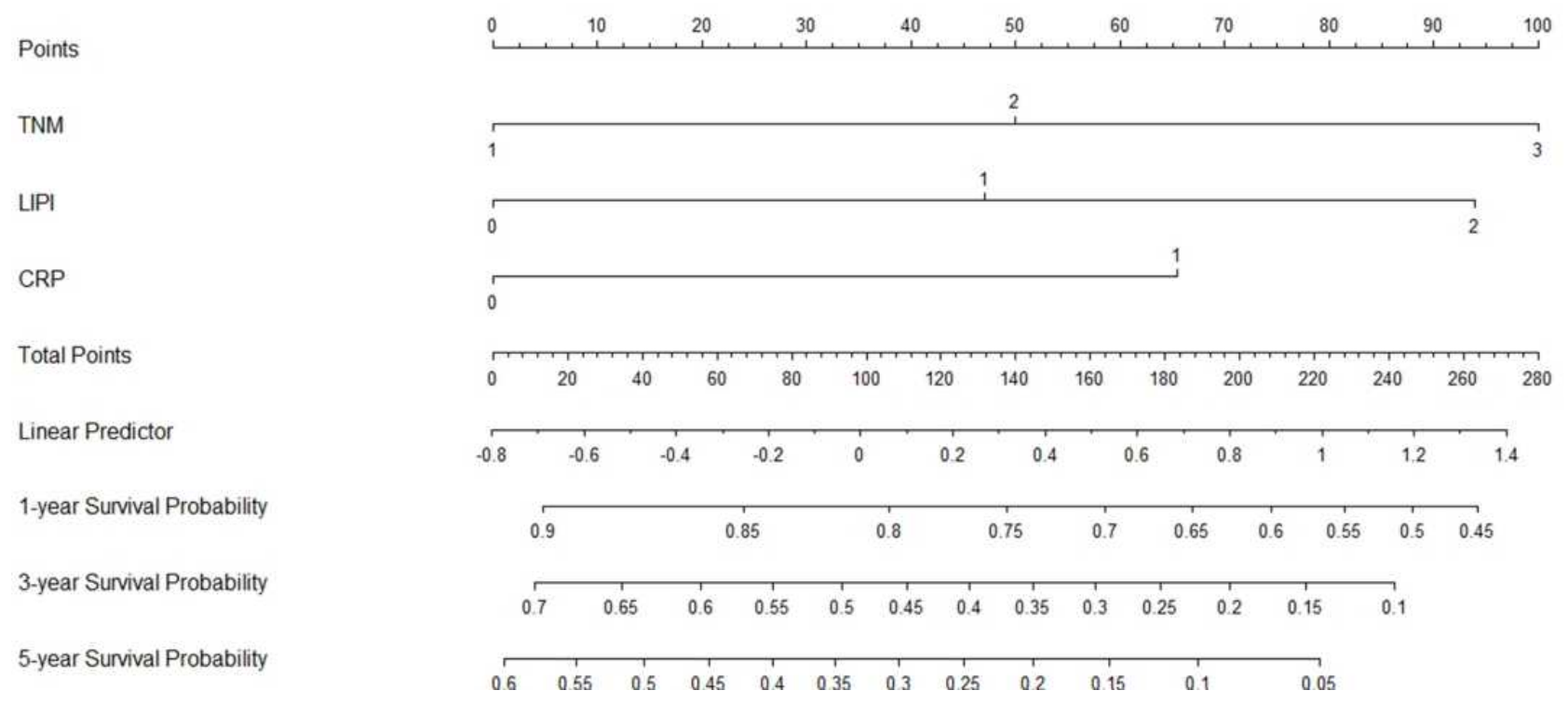

Figure 3 Prediction nomogram for CSS.

Notes: A nomogram, including LIPI, TNM and CRP, was conducted to predict the CSS probability in ESCC. Points of the factors attributed to an individual patient were located on each variable axis. The total points for all factors located on the total point axis were added up to indicate the probability of I-, 3- or 5-year CSS.

Abbreviations: ESCC, esophageal squamous cell carcinoma; LIPI, lung immune prognostic index; CRP, C-reactive protein (0: $\leq 10 \mathrm{mg} / \mathrm{L}$ and I: $>10 \mathrm{mg} / \mathrm{L})$; CSS, cancerspecific survival; TNM, tumor node metastasis (I: TNM I; 2: TNM II; 3: TNM III).

we did not put the ALB included into the nomogram because ALB was not an independent prognostic factor according to the multivariate analyses.

A previous published study reported that LIPI, a prognostic index combined with inflammatory biomarkers, has important clinical implications in patients treated with immune checkpoint inhibitors. ${ }^{18}$ Moreover, the potential bias could be decreased by the combination of dNLR and LDH because every single index might be affected by various factors. In the current study, it is worth noting that LIPI can be considered with potential applications in the clinical practice in patients with resected ESCC. The high score of LIPI was associated with inflammation, lymphatic metastasis and advanced TNM stage. Therefore, if a patient with high score of LIPI, it is recommended to improve the status of inflammation before surgery, or to perform adjuvant therapy after surgery. The validity of LIPI needs to be expected with more randomized prospective trials in the future.

The study has some limitations. Firstly, this study is mainly limited due to a single-center study with the retrospective character. Secondly, patients in the current study were treated without any preoperative therapy, the applications should be limited. Thirdly, the validity of LIPI needs to be expected with more large-sample, double-blinded, randomized prospective trials in the future.

\section{Conclusion}

In summary, the present study is the first study to determine the association between LIPI (based on LDH and dNLR) and prognosis in extra-pulmonary cancer. We confirmed that the LIPI is still an independent prognostic marker in patients with resected ESCC.

\section{Acknowledgments}

This study was supported by grants from Zhejiang Medical and Health Science and Technology Project (2018KY290, 2019RC129). This study was also supported by Zhejiang TCM Science and Technology Project (2021ZB034).

\section{Disclosure}

The authors declare that they have no competing interests.

\section{References}

1. Lin Y, Totsuka Y, He Y, et al. Epidemiology of esophageal cancer in Japan and China. J Epidemiol. 2013;23(3):233-242. doi:10.2188/jea. JE20120162

2. Chen W, Zheng R, Baade PD, et al. Cancer statistics in China, 2015. CA Cancer J Clin. 2016;66(2):115-132. doi:10.3322/caac.21338

3. Wang Y, Zhu L, Xia W, Wang F. Anatomy of lymphatic drainage of the esophagus and lymph node metastasis of thoracic esophageal cancer. Cancer Manag Res. 2018;10:6295-6303. doi:10.2147/ CMAR.S182436 
4. Feng JF, Sheng C, Zhao Q, Chen P. Prognostic value of mean platelet volume/platelet count ratio in patients with resectable esophageal squamous cell carcinoma: a retrospective study. PeerJ. 2019;7: e7246. doi:10.7717/peerj.7246

5. Mantovani A, Allavena P, Sica A, Balkwill F. Cancer-related inflammation. Nature. 2018;454(7203):436-444. doi:10.1038/ nature07205

6. Sun Y, Zhang L. The clinical use of pretreatment NLR, PLR, and LMR in patients with esophageal squamous cell carcinoma: evidence from a meta-analysis. Cancer Manag Res. 2018;10:6167-6179. doi:10.2147/CMAR.S171035

7. $\mathrm{Wu} \mathrm{CC}, \mathrm{Li} \mathrm{SH}, \mathrm{Lu} \mathrm{HI}$, et al. Inflammation-based prognostic scores predict the prognosis of locally advanced cervical esophageal squamous cell carcinoma patients receiving curative concurrent chemoradiotherapy: a propensity score-matched analysis. PeerJ. 2018;6: e5655. doi:10.7717/peerj.5655

8. Chen MF, Chen PT, Kuan FC, Chen WC. The predictive value of pretreatment neutrophil-to-lymphocyte ratio in esophageal squamous cell carcinoma. Ann Surg Oncol. 2019;26(1):190-199. doi:10.1245/ s10434-018-6944-1

9. Ren K, Yin Y, He F, Shao Y, Wang S. Prognostic role of derived neutrophil-to-lymphocyte ratio in surgical triple-negative breast cancer Cancer Manag Res. 2018;10:4891-4898. doi:10.2147/CMAR.S180695

10. Gong T, Liu J, Jiang J, et al. The role of lactate deshydrogenase levels on non-small cell lung cancer prognosis: a meta-analysis. Cell Mol Biol. 2019;65(1):89-93. doi:10.14715/cmb/2019.65.1.16

11. Yu SL, Xu LT, Qi Q, et al. Serum lactate dehydrogenase predicts prognosis and correlates with systemic inflammatory response in patients with advanced pancreatic cancer after gemcitabine-based chemotherapy. Sci Rep. 2017;7:45194. doi:10.1038/srep45194

12. Kiba T, Ito T, Nakashima T, et al. Bortezomib and dexamethasone for multiple myeloma: higher AST and LDH levels associated with a worse prognosis on overall survival. BMC Cancer. 2014;14 (1):462. doi:10.1186/1471-2407-14-462

13. Chen R, Zhou X, Yu Z, Liu J, Huang G. Low expression of LDHB correlates with unfavorable survival in hepatocellular carcinoma: strobe-compliant article. Medicine. 2015;94(39):e1583. doi:10.1097/ MD.0000000000001583

14. Schmidt H, Suciu S, Punt CJ, et al. Pretreatment levels of peripheral neutrophils and leukocytes as independent predictors of overall survival in patients with American Joint Committee on Cancer Stage IV Melanoma: results of the EORTC 18951 biochemotherapy trial. J Clin Oncol. 2007;25(12):1562-1569. doi:10.1200/JCO.2006.09.0274

15. Wei XL, Zhang DS, He MM, et al. The predictive value of alkaline phosphatase and lactate dehydrogenase for overall survival in patients with esophageal squamous cell carcinoma. Tumour Biol. 2016;37 (2):1879-1887. doi:10.1007/s13277-015-3851-y

16. Zhang P, Xi M, Li QQ, et al. The modified Glasgow prognostic score is an independent prognostic factor in patients with inoperable thoracic esophageal squamous cell carcinoma undergoing chemoradiotherapy. $J$ Cancer. 2014;5(8):689-695. doi:10.7150/ jca.9569
17. Huang H, Wang XP, Li XH, et al. Prognostic value of pretreatment alanine aminotransferase/aspartate aminotransferase (ALT/AST) ratio and gamma glutamyltransferase (GGT) in patients with esophageal squamous cell carcinoma. BMC Cancer. 2017;17:544. doi:10.1186/ s12885-017-3523-y

18. Mezquita L, Auclin E, Ferrara R, et al. Association of the lung immune prognostic index with immune checkpoint inhibitor outcomes in patients with advanced non-small cell lung cancer. JAMA Oncol. 2018;4(3):351-357. doi:10.1001/jamaoncol.2017.4771

19. Minami S, Ihara S, Komuta K. Pretreatment lung immune prognostic index is a prognostic marker of chemotherapy and epidermal growth factor receptor tyrosine kinase inhibitor. World J Oncol. 2019;10 (1):35-45. doi:10.14740/wjon1179

20. Kazandjian D, Gong Y, Keegan P, Pazdur R, Blumenthal GM Prognostic value of the lung immune prognostic index for patients treated for metastatic non-small cell lung cancer. JAMA Oncol. 2019;5(10):1481-1485. doi:10.1001/jamaoncol.2019.1747

21. Sonehara K, Tateishi K, Komatsu M, Yamamoto H, Hanaoka M. Lung immune prognostic index as a prognostic factor in patients with small cell lung cancer. Thorac Cancer. 2020;11(6):1578-1586. doi:10.1111/1759-7714.13432

22. Sorich MJ, Rowland A, Karapetis CS, Hopkins AM. Evaluation of the lung immune prognostic index for prediction of survival and response in patients treated with atezolizumab for non-small cell lung cancer: pooled analysis of clinical trials. J Thorac Oncol. 2019;14(8):1440-1446. doi:10.1016/j.jtho.2019.04.006

23. Rice TW, Rusch VW, Ishwaran H, Blackstone EH, Worldwide Esophageal Cancer Collaboration. Cancer of the esophagus and esophagogastric junction: data-driven staging for the seventh edition of the American Joint Committee on Cancer/International Union against cancer staging manuals. Cancer. 2010;116(16):3763-3773. doi: $10.1002 /$ cncr. 25146

24. Iasonos A, Schrag D, Raj GV, Panageas KS. How to build and interpret a nomogram for cancer prognosis. J Clin Oncol. 2008;26 (8):1364-1370. doi:10.1200/JCO.2007.12.9791

25. Su D, Zhou X, Chen Q, et al. Prognostic nomogram for thoracic esophageal squamous cell carcinoma after radical esophagectomy. PLoS One. 2015;10(4):e0124437. doi:10.1371/journal.pone.0124437

26. Zhang H, Guo XW, Yin XX, Liu YC, Ji SJ. Nomogram-integrated c-reactive protein/albumin ratio predicts efficacy and prognosis in patients with thoracic esophageal squamous cell carcinoma receiving chemoradiotherapy. Cancer Manag Res. 2019;11:9459-9468. doi:10.2147/CMAR.S228113

27. Shao Y, Ning Z, Chen J, et al. Prognostic nomogram integrated systemic inflammation score for patients with esophageal squamous cell carcinoma undergoing radical esophagectomy. Sci Rep. 2015;5:18811. doi:10.1038/srep18811
Cancer Management and Research is an international, peer-reviewed open access journal focusing on cancer research and the optimal use of preventative and integrated treatment interventions to achieve improved outcomes, enhanced survival and quality of life for the cancer patient.
The manuscript management system is completely online and includes a very quick and fair peer-review system, which is all easy to use. Visit http://www.dovepress.com/testimonials.php to read real quotes from published authors. 